\title{
Diagnostics for latent TB infection: incremental, not transformative progress
}

\author{
Madhukar Pai ${ }^{1}$ and Giovanni Sotgiu ${ }^{2}$
}

Affiliations: ${ }^{1}$ McGill International TB Centre \& McGill Global Health Programs, McGill University, Montreal, QC, Canada. ${ }^{2}$ Clinical Epidemiology and Medical Statistics Unit, Dept of Biomedical Sciences, University of Sassari, Sassari, Italy.

Correspondence: Giovanni Sotgiu, Clinical Epidemiology and Medical Statistics Unit, Dept of Biomedical Sciences, University of Sassari, Sassari, Italy. E-mail: gsotgiuduniss.it

0 @ERSpublications

Existing latent TB tests have limited predictive value; transformative progress is needed to develop better tools http://ow.ly/W4m4k

Diagnosis and treatment of latent tuberculosis infection (LTBI) is one of the strategies recommended by the World Health Organization (WHO) to control and eliminate tuberculosis (TB) disease worldwide, and is one of the elements of the WHO End TB Strategy [1]. While several developed countries have implemented and scaled-up programmes to detect and treat LTBI, developing countries have mostly focused on active TB disease control (i.e. passive case finding and treatment of smear-confirmed patients).

With the recent publication of the WHO guidelines for the programmatic management of LTBI in 2014, resource-limited countries now have a clear strategy for implementing targeted LTBI diagnosis and treatment, specifically in key, high-risk populations, such as people living with HIV/AIDS, adult and child contacts of pulmonary TB cases, patients initiating anti-tumour necrosis factor therapy, patients with end-stage renal failure on dialysis, patients preparing for organ or haematological transplantation, and persons with silicosis $[2,3]$. The WHO guidelines also make conditional recommendations for systematic LTBI testing and treatment in other vulnerable subgroups, including illicit drug users, prison inmates, homeless people, migrants from high-incidence countries and healthcare workers.

With regard to acceptable methods of LTBI diagnosis, the WHO guidelines recommend that either a tuberculin skin test (TST) or an interferon- $\gamma$ release assay (IGRA) should be used to detect LTBI in high-income and upper middle-income countries with estimated TB incidences <100 per 100000 population (currently, 113 countries globally) [3]. TST is preferred and IGRA should not replace TST in low- and other middle-income countries whose TB incidence is $\geqslant 100$ per 100000 population $[2,3]$.

Thus, in low-resource settings, the century-old TST, using purified protein derivative (PPD) as the antigen, continues to be the mainstay of LTBI screening and, thus, the main driver of LTBI therapy. IGRAs are expensive to implement at scale in such settings. While the TST has several advantages in low-resource settings, including low reagent cost, no equipment costs, limited skill requirement and no requirement for laboratories, it does suffer from two major limitations. First, the specificity of PPD-based TST is compromised by late (i.e. post-infancy) or repeated bacille Calmette-Guerin (BCG) vaccination (i.e. booster vaccinations), and, to a limited extent, by exposure to non-tuberculous mycobacteria [4]. The second limitation is the limited predictive value for TB disease [5]. In other words, a majority of individuals with positive TST results do not progress to active TB disease.

A decade ago, IGRAs entered the scene, offering hope that the TST could be replaced by an in vitro assay with superior performance [6]. A decade later, after hundreds of research studies, we can state that both TST and IGRA are acceptable but imperfect diagnostic tests [5]. They are indirect markers of Mycobacterium tuberculosis exposure and neither test is able to accurately differentiate between LTBI and active TB [7], or resolve the various stages within the spectrum of M. tuberculosis infection [5, 8].

Received: Nov 162015 | Accepted: Nov 172015

Conflict of interest: Disclosures can be found alongside the online version of this article at erj.ersjournals.com

Copyright OERS 2016 
Both the TST and IGRAs cannot distinguish individuals who have successfully cleared $M$. tuberculosis infection from those who have true infection (that is amenable to therapy) [9]. This inability to differentiate can favour the administration of LTBI treatment in those without the infection and, thereby, increase costs and adverse events. Both the TST and IGRA have reduced sensitivity in immunocompromised patients, particularly in those with a severe immune depression, and have a low predictive value for progression to active TB $[5,10]$. As in PPD-based TST, a majority of individuals with positive IGRA results do not progress to active TB disease.

Compared with the PPD-based TST, IGRAs have overcome the limited specificity problem, as BCG vaccination does not impact the test results, but they have not overcome the problem of limited predictive value [10], which could have allowed a more tailored LTBI treatment strategy.

In this context, what is the role of newer, more specific skin tests for LTBI? C-Tb (Statens Serum Institute, Copenhagen, Denmark), a novel $M$. tuberculosis-specific skin test, containing early secreted antigenic target (ESAT)-6 and culture filtrate protein (CFP)-10 antigens, is one such product [11, 12]. Another product, Diaskintest (Generium Pharmaceutical, Moscow, Russia), is available commercially in Russia, Kazakhstan and Ukraine [13], while an ESAT-6 based Chinese skin test has undergone phase II trials [14].

By substituting PPD with M. tuberculosis-specific antigens, these newer skin tests appear to overcome the limitations of the PPD-based TST, as far as specificity is concerned $[11,12]$. But do they offer a reasonably high sensitivity? The study by Hoff et al. [15] in this issue of the European Respiratory Journal reports the results of a clinical trial to determine the sensitivity of the $\mathrm{C}$ - $\mathrm{Tb}$ skin test in a population of microbiologically confirmed TB cases in Cape Town, South Africa. This well-designed trial showed that the $\mathrm{C}-\mathrm{Tb}$ test was safe to use, and, with the lowest cut-off of 5-mm induration, had a sensitivity of $74 \%$, which was comparable to that of QuantiFERON-TB Gold In Tube (Cellestis, Valencia, CA, USA) (75\%). The PPD-based TST, however, had the highest sensitivity of $88-90 \%$ in this population, depending on the cut-off used. The sensitivity of all tests was compromised in HIV-infected individuals with severe immunosuppression.

TABLE 1 A comparison of available diagnostics for latent tuberculosis (TB) infection

\begin{tabular}{|c|c|c|c|}
\hline & $\begin{array}{l}\text { PPD-based tuberculin skin } \\
\text { tests }\end{array}$ & $\begin{array}{l}\text { Newer, specific } \\
\text { skin tests }\end{array}$ & $\begin{array}{l}\text { IFN- } \gamma \text { release } \\
\text { assays }\end{array}$ \\
\hline Examples of products in the category & Tubersol, Aplisol, PPD RT23 & C-Tb, Diaskintest & $\begin{array}{l}\text { QuantiFERON-TB Gold } \\
\text { In Tube, T-SPOT.TB }\end{array}$ \\
\hline Testing format & Intra-dermal skin test (in vivo) & Intra-dermal skin test (in vivo) & $\begin{array}{c}\text { Ex vivo assay (ELISA or } \\
\text { ELISPOT) }\end{array}$ \\
\hline Antigens used & PPD & ESAT -6 and CFP-10 & ESAT-6 and CFP-10 \\
\hline In immunocompromised populations & Reduced & Reduced & Reduced \\
\hline Specificity & Modest & High & High \\
\hline Impact of BCG & $\begin{array}{l}\text { High (when BCG is given after } \\
\text { infancy or multiple times) }\end{array}$ & None & None \\
\hline Ability to distinguish latent from active TB & Low & Low & Low \\
\hline $\begin{array}{l}\text { Ability to predict progression to } \\
\text { active TB disease }\end{array}$ & Modest & $\begin{array}{l}\text { Unknown (but likely to be } \\
\text { modest based on indirect } \\
\text { evidence from IGRAs) }\end{array}$ & Modest \\
\hline Reagent costs & Low & $\begin{array}{l}\text { Unknown (but likely to be low } \\
\text { based on indirect evidence } \\
\text { from PPD-based TST) }\end{array}$ & High \\
\hline Requirement for laboratories & No & No & Yes \\
\hline
\end{tabular}

Manufacturer details for the products mentioned are as follows. Tubersol: Sanofi Pasteur Ltd, Toronto, ON, Canada. Aplisol: Parkedale Pharmaceuticals Inc., Rochester, MI, USA. PPD RT23: Statens Serum Institute, Copenhagen, Denmark. C-Tb: Statens Serum Institute, Copenhagen, Denmark. Diaskintest: Generium Pharmaceutical, Moscow, Russia. QuantiFERON-TB Gold In Tube: Cellestis, Valencia, CA, USA. T-SPOT.TB: Oxford Immunotec Global PLC, Abingdon, UK. PPD: purified protein derivative; IFN: interferon; ESAT: early secreted antigen target; CFP: culture filtrate protein; LTBI: latent tuberculosis infection; BCG: bacille Calmette-Guerin; IGRA: interferon- $\gamma$ release assay; M. tuberculosis: Mycobacterium tuberculosis; TST: tuberculin skin test. 
Acknowledging the limited evidence base, it appears that newer skin tests do offer higher specificity than PPD-based TST, but this might come at the cost of sensitivity (table 1). As the authors acknowledge, while there are no data on the predictive value of the newer skin tests, it is highly likely that the predictive value will be modest, based on what we know about IGRAs that use ESAT-6 and CFP-10 antigens [10].

In summary, when compared with the PPD-based TST, IGRAs and newer skin tests might offer some incremental advantages, primarily improved specificity (table 1). Newer skin tests, if reagents can be produced at scale, may also offer a system to resolve PPD shortages, which are becoming increasingly frequent [16], including in high-income countries. But sadly, none of the available LTBI diagnostics really get us what we want: a highly predictive test that can help target those who will benefit most from LTBI therapy. To develop such predictive tests, we need transformative research that will enable us to identify biomarkers or bio-signatures that can resolve the LTBI spectrum [8] and help target those at highest risk of progressing to active disease.

Target product profiles for such a transformative LTBI test are being developed by the Foundation for Innovative New Diagnostics (FIND) (Geneva, Switzerland), the New Diagnostics Working Group of the Stop TB Partnership, WHO, and other partners. Recent TB diagnostic market analyses and projections might also help increase industry and donor interest in research and development [17], resulting in the development of much-needed innovative products. It is important that future tools are validated not just for accuracy, but also for clinical impact, cost-effectiveness and feasibility in low-resource settings [18]. Of course, affordability of new tools is a critical consideration for scale-up and impact [18].

Until we have substantially improved tools to maximise the predictive value of existing tests, LTBI screening should be reserved for those who are at a sufficiently high risk of progressing to disease. Such high-risk individuals may be identifiable using multivariable risk prediction models, which incorporate test results with traditional risk factors, and using serial testing to resolve underlying phenotypes [5].

\section{References}

1 World Health Organization. The End TB Strategy. Global Strategy and Targets for Tuberculosis Prevention, Care and Control after 2015. www.who.int/tb/post2015_TBstrategy.pdf?ua=1 Date last accessed: April 7, 2015. Date last updated: November 2015.

2 World Health Organization. Guidelines on the management of latent tuberculosis infection. WHO/HTM/TB/ 2015.01. Geneva, WHO, 2014.

3 Getahun H, Matteelli A, Abubakar I, et al. Management of latent Mycobacterium tuberculosis infection: WHO guidelines for low tuberculosis burden countries. Eur Respir J 2015; 46: 1563-1576.

4 Farhat M, Greenaway C, Pai M, et al. False-positive tuberculin skin tests: what is the absolute effect of BCG and non-tuberculous mycobacteria? Int J Tuberc Lung Dis 2006; 10: 1192-1204.

5 Pai M, Denkinger CM, Kik SV, et al. Gamma interferon release assays for detection of Mycobacterium tuberculosis infection. Clin Microbiol Rev 2014; 27: 3-20.

6 Pai M, Riley LW, Colford JM Jr. Interferon-gamma assays in the immunodiagnosis of tuberculosis: a systematic review. Lancet Infect Dis 2004; 4: 761-776.

7 Sester M, Sotgiu G, Lange C, et al. Interferon-gamma release assays for the diagnosis of active tuberculosis: a systematic review and meta-analysis. Eur Respir J 2011; 37: 100-111.

8 Barry CE III, Boshoff HI, Dartois V, et al. The spectrum of latent tuberculosis: rethinking the biology and intervention strategies. Nat Rev Microbiol 2009; 7: 845-855.

9 Mack U, Migliori GB, Sester M, et al. LTBI: latent tuberculosis infection or lasting immune responses to M. tuberculosis? A TBNET consensus statement. Eur Respir J 2009; 33: 956-973.

10 Rangaka MX, Wilkinson KA, Glynn JR, et al. Predictive value of interferon-gamma release assays for incident active tuberculosis: a systematic review and meta-analysis. Lancet Infect Dis 2012; 12: 45-55.

11 Aggerbeck H, Giemza R, Joshi P, et al. Randomised clinical trial investigating the specificity of a novel skin test (C-Tb) for diagnosis of M. tuberculosis infection. PLoS One 2013; 8: e64215.

12 Bergstedt W, Tingskov PN, Thierry-Carstensen B, et al. First-in-man open clinical trial of a combined rdESAT-6 and rCFP-10 tuberculosis specific skin test reagent. PLoS One 2010; 5: e11277.

13 Kiselev VI, Baranovskii PM, Rudykh IV, et al. [Clinical trials of the new skin test Diaskintest for the diagnosis of tuberculosis]. Problemy tuberkuleza i boleznei legkikh 2009; 2: 11-16.

14 Sun QF, Xu M, Wu JG, et al. Efficacy and safety of recombinant Mycobacterium tuberculosis ESAT-6 protein for diagnosis of pulmonary tuberculosis: a phase II trial. Med Sci Monit 2013; 19: 969-977.

15 Hoff ST, Peter JG, Theron G, et al. Sensitivity of C-Tb: a novel RD-1-specific skin test for the diagnosis of tuberculosis infection. Eur Respir J 2016; 47: 919-928.

16 Centers for Disease Control and Prevention. Extent and effects of recurrent shortages of purified-protein derivative tuberculin skin test antigen solutions - United States, 2013. MMWR Morb Mortal Wkly Rep 2013; 62: $1014-1015$.

17 TB Diagnostics Market in Select High-Burden Countries: Current Market and Future Opportunities for Novel Diagnostics. http://unitaid.org/images/marketdynamics/publications/TB_Diagnostics_Market_in_Select_High-Burden_ Countries_Current_Market_and_Future_Opportunities_for_Novel_Diagnostics.pdf Date last accessed: November 2, 2015. Date last updated: July 2015.

18 Hanson C, Sotgiu G, Loddenkemper R. Ensuring that the diagnosis of tuberculosis accelerates progress towards the Millennium Development Goals. Eur Respir J 2014; 44: 1-4. 